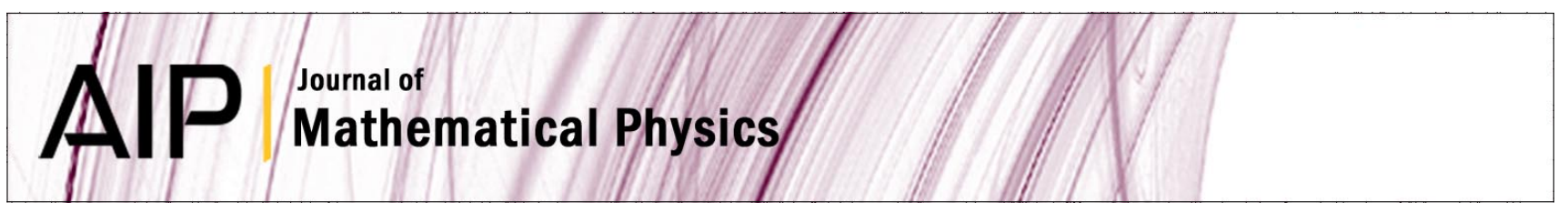

\title{
Path integrals evaluation in two-dimensional de Sitter space
}

A. V. Grinchuk and E. A. Ushakov

Citation: J. Math. Phys. 44, 146 (2003); doi: 10.1063/1.1526452

View online: http://dx.doi.org/10.1063/1.1526452

View Table of Contents: http://jmp.aip.org/resource/1/JMAPAQ/v44/i1

Published by the AIP Publishing LLC.

\section{Additional information on J. Math. Phys.}

Journal Homepage: http://jmp.aip.org/

Journal Information: http://jmp.aip.org/about/about_the_journal

Top downloads: http://jmp.aip.org/features/most_downloaded

Information for Authors: http://jmp.aip.org/authors

\section{ADVERTISEMENT}

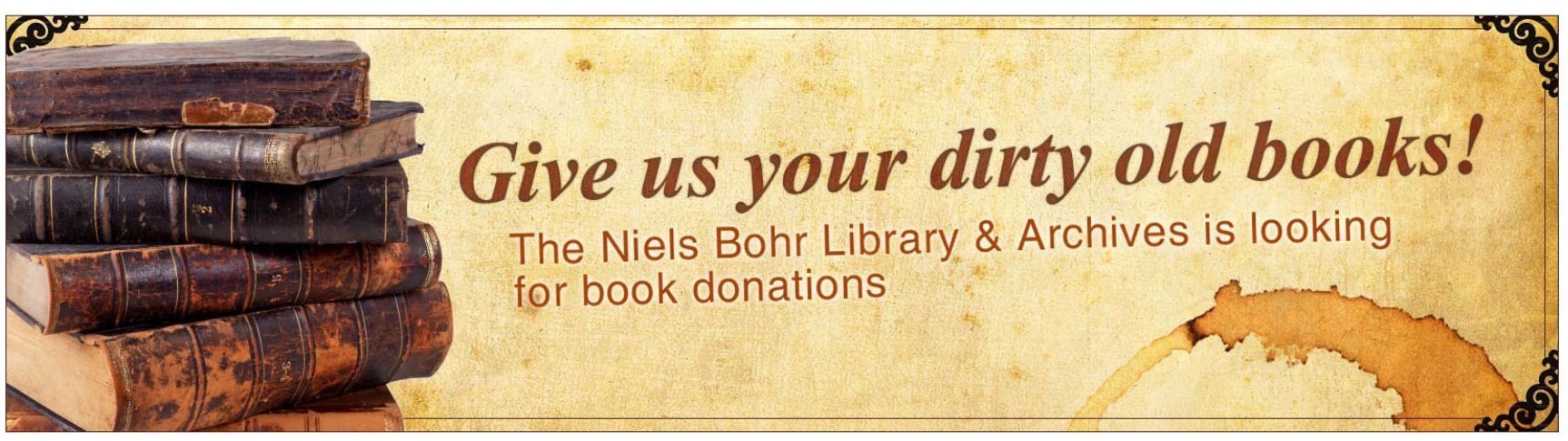




\section{Path integrals evaluation in two-dimensional de Sitter space}

A. V. Grinchuk and E. A. Ushakov

Belarussian State University, National Institute for Higher Education, Moskovskaya Str. 15, 521, 220001 Minsk, Belarus

(Received 8 June 2002; accepted 2 October 2002)

The propagator in de Sitter space is calculated based on the path integrals. The method of evaluation of path integrals for particles with spin is proposed. The calculations are compared with the quantum mechanical ones. () 2003 American Institute of Physics. [DOI: 10.1063/1.1526452]

\section{INTRODUCTION}

de Sitter space-time plays a special role in the general relativity theory (GRT): first, this space is curved, and, second, it possesses a maximal symmetry group. ${ }^{1}$ Therefore, there is a possibility to investigate the gravitational effect on quantum processes on the basis of exact analytical expressions.

The propagator is one of the major objects of a quantum field theory. By means of the propagator one can obtain a vacuum expectation value of stress-energy tensor, pair creation intensity, etc. Moreover, propagator plays a central role in the renormalization procedure in curved space-time. ${ }^{2}$

In this article it is offered to use the Feynman path integrals and proper time formalism ${ }^{3}$ for calculation of a propagator. Such an approach gives clear and simple interpretation of the process of a quantum particle interaction with an external gravitational field. In Ref. 4 the methods of a perturbation theory were used. However, in symmetric spaces the exact solution might be expected. Recent developments of the path integration technique actually enable one to solve this problem for a scalar particle. ${ }^{5}$

The Feynman integral for a particle with spin requires the operator of parallel transport, considerably complicating calculation of a propagator. In Refs. 6 and 7 the computational method suitable for two-dimensional spaces was proposed. In this case parallel transport is expressed through a phase factor, because the rotation group in two-dimensional space is Abelian. At the same time these methods fail in higher dimensional spaces. Moreover, we are unable to use directly the formalism developed in Refs. 5 and 8 because GRT deals with a wider class of manifolds than nonrelativistic quantum mechanics.

\section{DESCRIPTION OF THE METHOD}

\section{A. Propagator for spinning particle}

If the particle is described by Klein-Gordon equation (hereafter $c=\hbar=1$ )

$$
\nabla_{\mu} \nabla^{\mu} \phi+m^{2} \phi=0
$$

then the equation for Feynman propagator has the form

$$
\nabla_{\mu} \nabla^{\mu} G\left(x, x_{0}\right)+m^{2} G\left(x, x_{0}\right)=\delta\left(x, x_{0}\right) .
$$

The following substitution makes possible going to the path integral formulation: ${ }^{9}$

$$
G\left(x, x_{0}\right)=\frac{i}{2} \int_{0}^{\infty} d \tau e^{-i m^{2} \tau / 2}\left\langle x, \tau \mid x_{0}, 0\right\rangle,
$$




$$
\begin{gathered}
i \frac{\partial}{\partial \tau}\left\langle x, \tau \mid x_{0}, 0\right\rangle=-\frac{1}{2} \nabla_{\mu} \nabla^{\mu}\left\langle x, \tau \mid x_{0}, 0\right\rangle, \\
\left\langle x, 0 \mid x_{0}, 0\right\rangle=\delta\left(x, x_{0}\right),
\end{gathered}
$$

because we obtain the Schrödinger-type equation

$$
K\left(x, x_{0} ; \tau\right)=\left\langle x, \tau \mid x_{0}, 0\right\rangle=\int \mathcal{D} x(\tau) \exp \left\{-\frac{i}{2} \int\left(g_{\mu \nu} \dot{x}^{\mu} \dot{x}^{\nu}+\frac{R}{3}\right) d \tau\right\} .
$$

The appearance and the role of an additional term $\sim R / 3$ is discussed in Refs. 4 and 9 . Another way to obtain path integral formulation is based on the following operator identity: ${ }^{9}$

$$
\begin{aligned}
\frac{1}{\hat{H}}=i \int_{0}^{\infty} e^{-i \hat{H} \tau} d \tau \Rightarrow G\left(x, x_{0}\right) & =\left\langle x\left|\frac{1}{\nabla_{\mu} \nabla^{\mu}+m^{2}}\right| x_{0}\right\rangle \\
& =\frac{1}{2}\left\langle x\left|\frac{1}{\frac{1}{2}\left(\nabla_{\mu} \nabla^{\mu}+m^{2}\right)}\right| x_{0}\right\rangle \\
& =\frac{i}{2} \int_{0}^{\infty} d \tau e^{-i m^{2} \tau / 2}\left\langle x, \tau \mid x_{0}, 0\right\rangle .
\end{aligned}
$$

This formalism allows us to obtain the generalization of (7):

$$
\frac{1}{\hat{H}}=\hat{f} \frac{1}{\hat{f} \hat{H} \hat{f}} \hat{f}=i \hat{f} \int_{0}^{\infty} e^{-i \hat{f} \hat{H} \hat{f} \tau} d \tau \hat{f}
$$

Further, we will use this transformation with $\hat{f}=f(t)=\alpha / t$.

By analogy, for the particle with spin we have

$$
\begin{gathered}
\left(\nabla_{\mu} \nabla^{\mu}+m^{2}\right) \psi^{A}=0, \\
i \frac{\partial}{\partial \tau} K_{B_{0}}^{A}\left(x, x_{0} ; \tau\right)=-\frac{1}{2} \nabla_{\mu} \nabla^{\mu} K_{B_{0}}^{A}\left(x, x_{0} ; \tau\right), \\
K_{B_{0}}^{A}\left(x, x_{0} ; 0\right)=\delta\left(x, x_{0}\right) \delta_{B}^{A},
\end{gathered}
$$

but the Feynman propagator assumes the form

$$
G^{A^{\prime \prime}}{ }_{B^{\prime}}\left(x^{\prime \prime}, x^{\prime}\right)=\int_{0}^{\infty} d \tau e^{-i m^{2} \tau / 2} \int \mathcal{D} x(\tau) \exp \left\{-\frac{i}{2} \int\left(g_{\mu \nu} \dot{x}^{\mu} \dot{x}^{\nu}+\frac{R}{3}\right) d \tau\right\} P^{A^{\prime \prime}}{ }_{B^{\prime}}(x(\tau)),
$$

where $P^{A^{\prime \prime}}{ }_{B^{\prime}}(x(\tau))$ is an operator of parallel transport. ${ }^{7,10}$ The integral of such type has a more complicated structure than the ordinary one. This fact is caused by the difficulties connected with calculations of $P^{A^{\prime \prime}}{ }_{B^{\prime}}(x(\tau))$. Fortunately, fast development of the path integration technique gives us possibility to solve a wide range of problems. ${ }^{8}$ The two-dimensional case is a good demonstration of these difficulties and also shows the possible way to overcome them.

\section{B. Generation of an additional term by spin}

Let us start with the consideration of parallel transport on pseudosphere. Then the metric in horicyclic coordinates has the form (the case $\alpha=1$ is considered in Refs. 11 and 12) 


$$
d s^{2}=\left(\frac{\alpha}{t}\right)^{2}\left(d t^{2}+d x^{2}\right)
$$

where parameter $\alpha$ is closely related to the curvature scalar $R$ :

$$
R=-\frac{2}{\alpha^{2}}, \quad \alpha=\text { const. }
$$

It is convenient to go from the natural basis

$$
e_{t}=\frac{\partial}{\partial t}, \quad e_{x}=\frac{\partial}{\partial x}
$$

to orthonormal one

$$
e_{t}=\frac{t}{\alpha} \frac{\partial}{\partial t}, \quad e_{x}=\frac{t}{\alpha} \frac{\partial}{\partial x}
$$

and correspondingly

$$
\mathbf{d} t, \mathbf{d} x \rightarrow e^{t}=\left(\frac{\alpha}{t}\right) \mathbf{d} t, e^{x}=\left(\frac{\alpha}{t}\right) \mathbf{d} x .
$$

From (16) one can easily calculate the connection

$$
\omega_{x}^{t}=-\omega_{x}^{t}=-\frac{1}{t} \mathbf{d} x
$$

Then

$$
\nabla_{x} e^{t}=\frac{1}{t} e^{x}, \quad \nabla_{x} e^{x}=-\frac{1}{t} e^{t}
$$

It is convenient to use the following complex combination of basis vectors (isotropic basis):

$$
\epsilon^{+1}=\frac{1}{\sqrt{2}}\left(e^{t}+i e^{x}\right), \quad \epsilon^{-1}=\frac{1}{\sqrt{2}}\left(e^{t}-i e^{x}\right),
$$

in this basis

$$
\begin{aligned}
& \nabla_{x} \epsilon^{+1}=\nabla_{x} \frac{1}{\sqrt{2}}\left(e^{t}+i e^{x}\right)=\frac{1}{t} \frac{1}{\sqrt{2}}\left(e^{x}-i e^{t}\right)=-i \frac{1}{t} \epsilon^{+1} \\
& \nabla_{x} \epsilon^{-1}=\quad=i \frac{1}{t} \epsilon^{-1} .
\end{aligned}
$$

Similarly,

$$
\epsilon_{+1}=\frac{1}{\sqrt{2}}\left(\frac{t}{\alpha} \frac{\partial}{\partial t}-i \frac{t}{\alpha} \frac{\partial}{\partial x}\right), \quad \epsilon_{-1}=\frac{1}{\sqrt{2}}\left(\frac{t}{\alpha} \frac{\partial}{\partial t}+i \frac{t}{\alpha} \frac{\partial}{\partial x}\right)
$$

Hence parallel transport reduces to the rotation; the angle of rotation is given by 


$$
\chi=-\int \frac{1}{t} d x
$$

Then it is possible to obtain explicitly the rotation associated with a loop $\Gamma$,

$$
\chi(\Gamma)=-\oint_{\Gamma} \frac{1}{t} d x=\int \frac{1}{t^{2}} d S
$$

with $d S=d t d x$ as an element of spanned surface.

In the general case a two-dimensional manifold has the only "degree of freedom": in a tangent Euclidean space $e_{i}^{2}=1, d e_{1,2} \sim e_{2,1} d x$-rotation in a single plane. Any geometrical object can be expanded into the sum of proper vectors of the operator of rotation:

$$
i \hat{\mathbf{L}} \epsilon^{s}=s \boldsymbol{\epsilon}^{s}, \quad s=0, \quad \pm \frac{1}{2}, \quad \pm 1, \ldots \ldots
$$

Then the law of parallel transports acquires the simplest form

$$
\hat{\mathrm{P}} \epsilon^{s}=e^{i s \chi} \epsilon^{s}=e^{-i s \int(1 / t) d x} \epsilon^{s}, \quad \nabla_{x} \epsilon^{s}=-i s \frac{1}{t} \epsilon^{s}
$$

Hence the path integral will have the form

$$
K\left(x, x_{0} ; \tau\right)=\sum_{s} \epsilon^{s}(x) \otimes \epsilon_{s}\left(x_{0}\right) \int \mathcal{D}\{x(\tau)\} e^{i S(x(\tau))+i s \chi(x(\tau))} .
$$

When the metric is pseudo-Euclidean, the isotropic basis looks like

$$
\epsilon^{ \pm 1}=\frac{1}{\sqrt{2}}\left(\vec{e}_{0} \pm \vec{e}_{1}\right)
$$

and parallel transport can be described by a real phase factor:

$$
\hat{\mathrm{P}} \epsilon^{s}=e^{s \chi} \epsilon^{s}
$$

Then

$$
e^{i S(x(t))} \rightarrow e^{i S(x(t))+i s \chi(x(t))}
$$

and additional term will be incorporated into Lagrangian in the following manner:

$$
\mathcal{L} \rightarrow \mathcal{L}_{\text {eff }}=\mathcal{L}-i s \omega_{i} \dot{x}^{i}
$$

\section{PARTICLE WITH SPIN ON PSEUDOSPHERE}

In the isotropic basis the calculation of the operator of parallel transport is reduced to calculation of a phase factor, and additional term arises in the effective Lagrangian:

$$
L_{\mathrm{eff}}=\frac{1}{2}\left(\frac{\alpha^{2}}{t^{2}}\left(\dot{t}^{2}+\dot{x}^{2}\right)-\frac{s}{t} \dot{x}\right)
$$

where $s$ is the spin weight. ${ }^{7}$ The problem is reduced to the quantum mechanical one with a Hamiltonian of the form

$$
H=\frac{t}{\alpha}\left(\frac{p_{t}^{2}+p_{x}^{2}}{2}-\frac{p_{x}}{t} s+\frac{s^{2}-m^{2} \alpha^{2}}{2 t^{2}}\right) \frac{t}{\alpha}
$$


Integration over $x$ is quite simple, because neither $L_{\text {eff }}$ nor $H$ depends on $x(t)$ explicitly, but only on $\dot{x}(t)$ and $p_{x}$. In this case

$$
\int \mathcal{D}(x(\tau)) \mathcal{D}\left(p_{x}(\tau)\right) e^{i \int p_{x} \dot{x} d \tau-i \int f\left(p_{x}(\tau)\right) d \tau}=\int d p_{x} e^{i p_{x}\left(x-x_{0}\right)-i f\left(p_{x}\right) \tau} .
$$

Using (7) one obtains

$$
\begin{aligned}
\frac{1}{H}= & \left\langle t^{\prime \prime}, x^{\prime \prime}\left|\frac{\alpha}{t^{\prime \prime}} \frac{1}{\left(p_{t}^{2}+p_{x}^{2}\right) / 2-\left(p_{x} / t\right) s+\left[\left(s^{2}-m^{2} \alpha^{2}\right) / 2 t^{2}\right]} \frac{\alpha}{t^{\prime}}\right| t^{\prime}, x^{\prime}\right\rangle \\
= & \frac{i}{2} \frac{\alpha^{2}}{t^{\prime \prime} t^{\prime}} \int_{0}^{\infty} d \tau \int d p_{x} \exp \left(i p_{x}^{2} \tau / 2\right) \exp \left(i p\left(x^{\prime \prime}-x^{\prime}\right)\right) \\
& \times \int \mathcal{D} t(\tau) \exp i \int d \tau\left(\frac{\dot{t}^{2}}{2}-\frac{p_{x}}{t} s+\frac{s^{2}-m^{2} \alpha^{2}}{2 t^{2}}\right) .
\end{aligned}
$$

When integrating over $t$, one deals with a radial Coulomb problem. Its solution by means of continual integration can be found in Ref. 13. The propagator can be written as decomposition by eigenfunctions:

$$
K\left(t^{\prime \prime}, x^{\prime \prime} ; t^{\prime}, x^{\prime} ; \tau\right)=\frac{\alpha^{2}}{t^{\prime \prime} t^{\prime}} \sum_{s} \epsilon^{s}(x) \otimes \epsilon_{s}\left(x_{0}\right) \int d p_{t} d p_{x} e^{i \tau\left(p_{t}^{2}+p_{x}^{2}\right) / 2} e^{i p_{x}\left(x^{\prime \prime}-x^{\prime}\right)}{ }_{s} \psi\left(p_{t}, t^{\prime \prime}\right){ }_{s} \psi^{*}\left(p_{t}, t^{\prime}\right),
$$

where

$$
\begin{gathered}
{ }_{s} \psi\left(p_{t}, t\right)=\frac{\Gamma\left(\frac{1}{2}+\nu-i s p_{x} / p_{t}\right)}{\sqrt{2 \pi} \Gamma(2 \nu+2)} M_{i s p_{x} / p_{t}, \nu}\left(-2 i p_{t} t\right), \\
{ }_{s} \psi^{*}\left(p_{t}, t\right)=\frac{\Gamma\left(\frac{1}{2}+\nu+i s p_{x} / p_{t}\right)}{\sqrt{2 \pi} \Gamma(2 \nu+2)} M_{-i s p_{x} / p_{t}, \nu}\left(2 i p_{t} t\right), \\
\nu=\sqrt{s^{2}-m^{2} \alpha^{2}+1 / 4},
\end{gathered}
$$

and $M_{s, \nu}(x)$ is the Whittaker function.

\section{TRANSITION TO de SITTER SPACE}

The above decompositions of the propagator (31) were obtained as solutions of quantum mechanical problems in polar coordinates. In order to go from the space with the metric

$$
d s^{2}=\left(\frac{\alpha}{t}\right)^{2}\left(d t^{2}+d x^{2}\right), \quad \text { with } t \geqslant 0, \quad-\infty \leqslant x \leqslant \infty
$$

to

$$
d s^{2}=\left(\frac{\alpha}{t}\right)^{2}\left(d t^{2}-d x^{2}\right), \quad \text { with }-\infty \leqslant t, x \leqslant \infty
$$

one has to take into account the change of the variable $t$ range and the change of the sign of the term $d x^{2}$. 
The following limitation has been implicitly used in a radial Coulomb problem: radial coordinate varies in the range $(0, \infty)$. This means the use of the mirror principle (see discussion of boundary conditions in Refs. 5 and 8):

$$
\psi(r) \rightarrow \psi(r)-\psi(-r) .
$$

In de Sitter space the appropriate coordinate varies from $-\infty$ to $\infty$. Therefore, it is necessary to discard the reflected part of a wave. It seems to be convenient to consider asymptotics of eigenfunctions, as at $t \rightarrow \infty$ the effective potential $\left(-i\left(p_{x} / t\right) s+\left(s^{2}-m^{2} \alpha^{2}\right) / 2 t^{2}\right)$ tends to zero, and eigenfunctions tends to $e^{ \pm i p_{t} t}$. For a scalar particle with

$$
\begin{gathered}
s=0, \\
M_{0, \nu}(2 i z)=i^{\nu+1 / 2} 2^{2 \nu+1 / 2} \sqrt{z} \Gamma(1+\nu) J_{\nu}(z),
\end{gathered}
$$

the decomposition by cylindrical Bessel functions with the asymptotic

$$
J_{n}(x) \sim \sqrt{\frac{2}{\pi x}} \sin \left(x-\frac{n \pi}{2}+\frac{\pi}{4}\right)=\sqrt{\frac{2}{\pi x}} \frac{1}{2 i}\left(e^{i(x-n \pi / 2+\pi / 4)}-e^{-i(x-n \pi / 2+\pi / 4)}\right)
$$

can be obtained.

Cylindrical Hankel functions have the required asymptotic:

$$
H_{n}^{(1)}(p t) \sim \sqrt{\frac{1}{p t}} e^{i p t}, \quad H_{n}^{(2)}(p t) \sim \sqrt{\frac{1}{p t}} e^{-i p t}
$$

The Bessel function represents their linear combination. Then for a temporal part of the propagator we receive decomposition

$$
K\left(t^{\prime \prime}, t^{\prime} ; s\right)=\int p_{t} d p_{t} \sqrt{t^{\prime \prime} t^{\prime}} H_{\nu}^{(1)}\left(p_{t} t^{\prime \prime}\right) H_{\nu}^{(1) *}\left(p_{t} t^{\prime}\right) e^{-i s p_{t}^{2} / 2}, \quad \nu=\sqrt{-m^{2} \alpha^{2}+1 / 4} .
$$

Similarly, for a particle with spin we should proceed from the Whittaker function of the first kind $M_{k, m}(x)$ to the Whittaker function of the second kind $W_{k, m}(x)$ :

$$
\begin{aligned}
W_{k, m}(x)= & \frac{\Gamma(-2 m)}{\Gamma\left(\frac{1}{2}-m-k\right)} x^{m+1 / 2} e^{-x / 2}{ }_{1} F_{1}\left(m+\frac{1}{2}-k ; 2 m+1 ; x\right) \\
& +\frac{\Gamma(2 m)}{\Gamma\left(\frac{1}{2}+m-k\right)} x^{-m+1 / 2} e^{-x / 2}{ }_{1} F_{1}\left(-m+\frac{1}{2}-k ;-2 m+1 ; x\right),
\end{aligned}
$$

possessing the asymptotics

$$
W_{k, m}(x) \sim x^{k} e^{-x / 2}, \quad x \rightarrow \pm \infty .
$$

The functions $M_{k, m}(x)$ represent a linear combination of $W_{k, m}(x)$ and $W_{-k, m}(-x)$.

The outcome can be again represented as decomposition by eigenfunctions:

$$
\begin{gathered}
K\left(t^{\prime \prime}, x^{\prime \prime} ; t^{\prime}, x^{\prime} ; \tau\right)=\frac{\alpha^{2}}{t^{\prime \prime} t^{\prime}} \sum_{s} \epsilon^{s}(x) \otimes \epsilon_{s}\left(x_{0}\right) \int d p_{t} d p_{x} e^{i \tau\left(p_{t}^{2}+p_{x}^{2}\right) / 2} e^{i p_{x}\left(x^{\prime \prime}-x^{\prime}\right)}{ }_{s} \psi\left(p_{t}, t^{\prime \prime}\right){ }_{s} \psi^{*}\left(p_{t}, t^{\prime}\right), \\
{ }_{s} \psi\left(t^{\prime \prime}\right)=e^{-i p_{x} \pi s / 2 p_{t}} W_{i s p_{x} / p_{t}, \nu}\left(-2 i p_{t} t\right), \quad \nu=\sqrt{s^{2}-m^{2} \alpha^{2}+\frac{1}{4}} .
\end{gathered}
$$

The second problem to be solved is the transition from pseudosphere metric 


$$
d s^{2}=\frac{\alpha^{2}}{t^{2}}\left(d t^{2}+d x^{2}\right)
$$

to the de Sitter space one

$$
d s^{2}=\frac{\alpha^{2}}{t^{2}}\left(d t^{2}-d x^{2}\right)
$$

It is convenient to rotate the $x$ axis: $x \rightarrow i x$, and correspondingly

$$
\begin{gathered}
p_{x} \rightarrow-i p_{x}, \\
\epsilon^{ \pm 1}=\frac{\alpha}{t} \frac{1}{\sqrt{2}}\left(e^{t \mp} e^{x}\right), \quad \epsilon_{ \pm 1}=\frac{1}{\sqrt{2}}\left(\frac{t}{\alpha} \frac{\partial}{\partial t} \pm \frac{t}{\alpha} \frac{\partial}{\partial x}\right), \\
\hat{P} \epsilon^{s}=e^{s \chi} \epsilon^{s}=e^{-s \int(1 / t) d x} \epsilon^{s}, \quad \nabla_{x} \epsilon^{s}=-s \frac{1}{t} \epsilon^{s}, \\
L_{\mathrm{eff}}=\frac{1}{2}\left(\frac{\alpha^{2}}{t^{2}}\left(\dot{t}^{2}-\dot{x}^{2}\right)-i \frac{s}{t} \dot{x}\right) .
\end{gathered}
$$

So, that propagator looks like

$$
\begin{aligned}
K\left(t^{\prime \prime}, x^{\prime \prime} ; t^{\prime}, x^{\prime} ; \tau\right)= & \frac{\alpha^{2}}{t^{\prime \prime} t^{\prime}} \sum_{s} \epsilon^{s} \otimes \epsilon_{s} e^{i \tau\left(p_{t}^{2}-p_{x}^{2}\right) / 2} \int d p_{t} d p_{x} e^{i p_{x} x^{\prime \prime}} e^{\pi p_{x} s / p_{t}} W_{p_{x} s / p_{t}, \nu}\left(2 i p_{t} t^{\prime \prime}\right) \\
& \times e^{-i p_{x} x^{\prime}} e^{\pi p_{x} s / p_{t}} W_{-p_{x} s / p_{t}, \nu}\left(-2 i p_{t} t^{\prime}\right)
\end{aligned}
$$

and

$$
G\left(t^{\prime \prime}, x^{\prime \prime} ; t^{\prime}, x^{\prime}\right)=\frac{i}{2} \int_{0}^{\infty} K\left(t^{\prime \prime}, x^{\prime \prime} ; t^{\prime}, x^{\prime} ; \tau\right) d \tau
$$

In order to make sure that this expression is a required propagator, one needs to analyze a short-time kernel. It is possible to use asymptotical expansion of Whittaker function

$$
\begin{aligned}
W_{a, c}(x) & \approx e^{-x / 2}\left(1-\frac{\left(\frac{1}{2}-a-c\right)\left(\frac{1}{2}-a+c\right)}{x}\right) x^{a} \\
& \approx \exp \left(-\left(\frac{\left(\frac{1}{2}-a-c\right)\left(\frac{1}{2}-a+c\right)}{x}\right)-\frac{x}{2}+a \ln (x)\right) .
\end{aligned}
$$

Substituting (38) into (36), one can ensure that

$$
\begin{gathered}
\int \frac{d p_{t}}{2 \pi} e^{\pi p_{x} s / p_{t}} W_{p_{x} s / p_{t}, \nu}\left(2 i p_{t} t^{\prime \prime}\right) e^{\pi p_{x} s / p_{t}} W_{-p_{x} s / p_{t}, \nu}\left(-2 i p_{t} t^{\prime}\right) \\
\rightarrow \frac{1}{\sqrt{2 \pi i \tau}} \exp \left(i \frac{\left(t^{\prime \prime}-t^{\prime}\right)^{2}}{2 \tau}-\frac{p_{x} s}{t^{\prime}} \tau+i \frac{\alpha^{2} m^{2}-s^{2}}{2 t^{\prime \prime} t^{\prime}} \tau\right)
\end{gathered}
$$

and this result actually leads to the initial effective Lagrangian (see the Appendix).

Propagator (36) has two important features:

(1) It satisfies the boundary conditions (11). Actually, if $\tau \rightarrow 0$, then 


$$
\frac{1}{\sqrt{2 \pi i \tau}} \exp \left(i \frac{\left(t^{\prime \prime}-t^{\prime}\right)^{2}}{2 \tau}-\frac{p_{x} s}{t^{\prime}} \tau+i \frac{\alpha^{2} m^{2}-s^{2}}{2 t^{\prime \prime} t^{\prime}} \tau\right) \rightarrow \delta\left(t^{\prime \prime}-t^{\prime}\right) .
$$

(2) This propagator satisfies Eq. (10) because eigenfunctions are the solutions of the equation

$$
-\frac{1}{2}\left(\nabla_{\mu} \nabla^{\mu}+m^{2}\right)\left(\epsilon^{s} e^{i p_{x} x^{\prime \prime}} \frac{\alpha}{t} W_{p_{x} s / p_{t}, \nu}\left(2 i p_{t} t^{\prime \prime}\right)\right)=\frac{p_{t}^{2}-p_{x}^{2}}{2}\left(\epsilon^{s} e^{i p_{x} x^{\prime \prime}} \frac{\alpha}{t} W_{p_{x} s / p_{t}, \nu}\left(2 i p_{t} t^{\prime \prime}\right)\right) .
$$

With (35) one can obtain

$$
\begin{gathered}
-\frac{d^{2}}{d t^{2}} W_{p_{x} s / p_{t}, \nu}\left(2 i p_{t} t^{\prime \prime}\right)-\left(\left(p_{x}+i \frac{s}{t}\right)^{2}+\frac{m^{2} \alpha^{2}}{t^{2}}\right) W_{p_{x} s / p_{t}, \nu}\left(2 i p_{t} t^{\prime \prime}\right)=\left(p_{t}^{2}-p_{x}^{2}\right) W_{p_{x} s / p_{t}, \nu}\left(2 i p_{t} t^{\prime \prime}\right), \\
-\frac{d^{2}}{d t^{2}} W_{p_{x} s / p_{t}, \nu}\left(2 i p_{t} t^{\prime \prime}\right)-\left(2 \frac{i s p_{x}}{t}+\frac{m^{2} \alpha^{2}-s^{2}}{t^{2}}\right) W_{p_{x} s / p_{t}, \nu}\left(2 i p_{t} t^{\prime \prime}\right)=p_{t}^{2} W_{p_{x} s / p_{t}, \nu}\left(2 i p_{t} t^{\prime \prime}\right) .
\end{gathered}
$$

The Whittaker function actually satisfies this equation.

It is interesting to analyze a special case of this solution. The vector particle propagator is expanded into wavefunctions of the form $\epsilon^{-1} W_{-1, \nu}(2 i k x)$ and $\epsilon^{+1} W_{1, \nu}(2 i k x)$, since after final integration only point $p_{t}^{2}-p_{x}^{2}=0$ will be taken into account and $s p_{x} / p_{t}= \pm 1$. It is common practice to solve equations for the vector field by separation into the transverse and longitudinal parts. Using the connection between Hankel and Whittaker functions,

$$
\begin{aligned}
H_{\nu}^{(1)}(-k t)=\sqrt{\frac{\pi}{2 i k t}} W_{0, \nu}(2 i k t) & =-\frac{1}{2(i k t)^{3 / 2}} \sqrt{\frac{2}{\pi}}\left(\left(\nu^{2}-\frac{1}{4}\right) W_{-1, \nu}(2 i k t)-W_{1, \nu}(2 i k t)\right), \\
H_{\nu+1}^{(1)}(-k t)+H_{\nu-1}^{(1)}(-k t)= & \frac{1}{2(i k t)^{3 / 2}} \sqrt{\frac{2}{\pi}}\left(\left(\nu^{2}-\frac{1}{4}\right) W_{-1, \nu}(2 i k t)+\left(W_{0, \nu}(2 i k t)\right.\right. \\
& \left.\left.+W_{1, \nu}(2 i k t)\right)\right),
\end{aligned}
$$

and the following relation

$$
\frac{d}{d t} H_{\nu}^{(1)}(-k t)=-\frac{i k}{2}\left(H_{\nu+1}^{(1)}(-k t)+H_{\nu-1}^{(1)}(-k t)\right),
$$

one can ensure that these parts represent a linear combination of the above solutions. Actually, the longitudinal one is

$$
\nabla\left(\sqrt{\frac{2 i k t}{\pi}} H_{\nu}^{(1)}(-k t) e^{i k x}\right)=\epsilon^{+1}\left(\nu^{2}-\frac{1}{4}\right) W_{-1, \nu}(2 i k t) e^{i k x}+\epsilon^{-1} W_{1, \nu}(2 i k t) e^{i k x} .
$$

The transverse part is proportional to

$$
\epsilon^{+1}\left(\nu^{2}-\frac{1}{4}\right) W_{-1, \nu}(2 i k t)-\epsilon^{-1} W_{1, \nu}(2 i k t) .
$$

\section{CONCLUSION}

The path integral for particles with spin includes an additional factor-the operator of parallel transport. The use of parallel transport requires the development of new calculation methods for functional integrals. In contrast to the differential equations approach, the path integral formalism 
deals with a global object - the propagator. It has more general analytical properties, and gives the possibility to distinguish connections between compact and noncompact spaces, and continuous and discrete spectrum.

Two-dimensional solutions have similar forms for arbitrary spin. A similar situation occurs also in some four-dimensional problems. Note the Teukolsky equation ${ }^{14}$ describing massless particles of spin $0, \frac{1}{2}, 1, \frac{3}{2}, 2$ in Kerr geometry.

Our interest in path integration is stimulated by the possibility of simple and clear description of a spinning quantum particle interaction with the curvature. ${ }^{15}$ This approach may be useful when obtaining common features of the fields of different spins in curved space and in quantization of the gravitational field itself.

\section{APPENDIX: PROOF OF EQ. (39)}

We will consider (39) with $\tau \rightarrow 0$. In this case

$$
p_{t} \rightarrow \frac{t^{\prime \prime}-t^{\prime} \tau \rightarrow 0}{\tau} \rightarrow \infty
$$

and one has to take into account terms up to $\left(t^{\prime \prime}-t^{\prime}\right)^{2}$. After the substitution of expansion

$$
\begin{aligned}
W_{a, c}(x) \stackrel{\substack{x \rightarrow \infty \\
\rightarrow}}{-x / 2}\left(1-\frac{\left(\frac{1}{2}-a-c\right)\left(\frac{1}{2}-a+c\right)}{x}+O\left(\frac{1}{x^{2}}\right)\right) x^{a} \\
\quad \approx \exp \left(-\left(\frac{\left.\left(\frac{1}{2}-a-c\right)\left(\frac{1}{2}-a+c\right)\right)}{x}\right)-\frac{x}{2}+a \ln (x)\right)
\end{aligned}
$$

to

$$
e^{-i p_{t}^{2} \tau} e^{\pi p_{x} s / p_{t}}\left(2 p_{t}\right)^{s p_{x} / p_{t}} W_{p_{x} s / p_{t}, \nu}\left(2 i p_{t} t^{\prime \prime}\right) e^{\pi p_{x} s / p_{t}}\left(2 p_{t}\right)^{-s p_{x} / p_{t}} W_{-p_{x} s / p_{t}, \nu}\left(-2 i p_{t} t^{\prime}\right)
$$

one has up to the accuracy of $1 / p_{t}^{2}$

$$
\begin{aligned}
& \exp \left(\frac{1}{p_{t}}\left(-\frac{i s^{2}}{2 t^{\prime \prime}}+\frac{i M^{2} \alpha^{2}}{2 t^{\prime \prime}}+p_{x} s \ln \left(2 i p_{t} t^{\prime \prime}\right)\right)-\frac{1}{p_{t}}\left(-\frac{i s^{2}}{2 t^{\prime}}+\frac{i M^{2} \alpha^{2}}{2 t^{\prime}}+p_{x} s \ln \left(2 i p_{t} t^{\prime}\right)\right)\right) e^{i p\left(t^{\prime \prime}-t^{\prime}\right)} \\
& =e^{i p\left(t^{\prime \prime}-t^{\prime}\right)} \exp \left(\frac{1}{p_{t}}\left(i\left(s^{2}+M^{2} \alpha^{2}\right)\left(\frac{1}{t^{\prime \prime}}-\frac{1}{t^{\prime}}\right)+s p_{x}\left(\ln \left(t^{\prime \prime}\right)-\ln \left(t^{\prime}\right)\right)\right)\right)
\end{aligned}
$$

After the use of

$$
\frac{f\left(t^{\prime \prime}\right)-f\left(t^{\prime}\right)}{p_{t}} \approx \tau \frac{f^{\prime}\left(t^{\prime}\right)\left(t^{\prime \prime}-t^{\prime}\right)+f^{\prime \prime}\left(t^{\prime}\right)\left(t^{\prime \prime}-t^{\prime}\right)^{2}+\cdots}{t^{\prime \prime}-t^{\prime}}=\tau f^{\prime}(t)+o(\tau),
$$

the following result can be obtained:

$$
\exp \left(i p_{t}\left(t^{\prime \prime}-t^{\prime}\right)-i \tau\left(p_{t}^{2}+i s \frac{p_{x}}{t^{\prime}}+\frac{s^{2}-M^{2} \alpha^{2}}{t^{\prime 2}}\right)\right)
$$

Finally, integration over $p_{t}$ can be made: 


$$
\begin{gathered}
\int_{-\infty}^{\infty} \exp \left(i p_{t}\left(t^{\prime \prime}-t^{\prime}\right)-i \tau\left(p_{t}^{2}+i s \frac{p_{x}}{t^{\prime}}+\frac{s^{2}-M^{2} \alpha^{2}}{t^{\prime 2}}\right)\right) \frac{d p_{t}}{2 \pi} \\
=\frac{1}{\sqrt{2 \pi i \tau}} \exp \left(i \frac{\left(t^{\prime \prime}-t^{\prime}\right)^{2}}{2 \tau}-\frac{p_{x} s}{t^{\prime}} \tau+i \frac{\alpha^{2} m^{2}-s^{2}}{2 t^{\prime \prime} t^{\prime}} \tau\right) .
\end{gathered}
$$

${ }^{1}$ E. G. Kalnins and W. Miller, Jr., J. Math. Phys. 15, 1263 (1974).

${ }^{2}$ N. Birrell and P. Devis, Quantum Field Theory in Curved Space - time (Cambridge University Press, Cambridge, 1984).

${ }^{3}$ V. A. Fock, Phys. Z. Sowjetunion 12, 404 (1937).

${ }^{4}$ J. D. Bekenstein and L. Parker, Phys. Rev. D 23, 2850 (1981).

${ }^{5}$ C. Grosche and F. Steiner, J. Math. Phys. 36, 2354 (1995).

${ }^{6}$ A. V. Grinchuk, Gravitation Electromagn. 6, 63 (1998) (in Russian).

${ }^{7}$ A. V. Grinchuk and E. A. Ushakov, Gravitation Cosmol. 5, 173 (1999).

${ }^{8}$ C. Grosche and F. Steiner, Handbook of Feynman Path Integrals (Springer-Verlag, Berlin, 1998).

${ }^{9}$ B. S. DeWitt, Rev. Mod. Phys. 29, 377 (1957).

${ }^{10}$ M. B. Mensky, Theor. Math. Phys. 18, 190 (1974) (in Russian).

${ }^{11}$ C. Grosche, Path Integrals, Hyperbolic Spaces, and Selberg Trace Formulae (World Scientific, Singapore, 1995).

${ }^{12}$ C. Grosche, "Path Integration and Separation of Variables In Spaces of Constant Curvature In Two And Three Dimensions," hep-th/9311001.

${ }^{13}$ F. Steiner, Phys. Lett. A 109, 363 (1984).

${ }^{14}$ S. Tenkolsky, Astrophys. J. 185, 635 (1973).

${ }^{15}$ E. A. Ushakov, Gravitation Electromagn. 6, 168 (1998) (in Russian). 